\title{
What do we know about tailoring treatment with tenofovir?
}

\author{
"Waiting for the results of prospective studies assessing the efficacy \\ and safety of TDF reduced doses when associated with protease \\ inhibitors (as recently envisaged) and for the long-term studies on TAF \\ safety and compartmental efficacy, a personalized approach to TDF \\ might be used."
}

First draft submitted: 4 January 2016; Accepted for publication: 4 January 2016; Published online: 4 April 2016

Keywords: HIV $\bullet$ pharmacogenetics $\bullet$ pharmacokinetics $\bullet$ renal $\bullet$ tenofovir $\bullet$ toxicity - tubular

\section{Tenofovir disoproxil fumarate \& renal toxicity}

Tenofovir disoproxil fumarate (TDF) is the prodrug of tenofovir (TFV), a nucleotide reverse transcriptase inhibitor active against HIV-1, HIV-2 and Hepatitis B virus (HBV). The compound is extensively used worldwide and over 7.5 million patient-years experience support its efficacy and tolerability [1]. Nevertheless TDF has been associated with incident chronic kidney disease, Fanconi's syndrome, subclinical tubular dysfunction, vitamin $\mathrm{D}$ deficiency and reduced bone mineral density [2]. Several strategies have been sought in order to avoid such adverse effects including switches to other compounds, regimen modifications and the development of a new prodrug (tenofovir alafenamide, TAF) that has shown comparable efficacy and significant tolerability improvements. TDF patent will soon expire and a better understanding of its actual mid- and long-term toxicity is critical in order to understand whether its use in specific circumstances and/or specific patients might be safe enough to allow for a likely cost reduction in the future.

TFV clearance occurs both by glomerular filtration and active tubular secretion: organic anion transporter 1, MRP-4 and MRP-7 have been found to be responsible of TFV uptake and extrusion by proximal tubular cells $[3,4]$. TFV accumulation inside proximal tubular cells causing subsequent mitochondrial damage has been postulated as the pathogenic mechanism and it has been confirmed in electronic microscopy studies [5].

\section{Factors involved in TDF-associated renal toxicity}

The reported serum creatinine elevation (and estimated glomerular filtration decrease) under TDF-based treatment might be related to the decrease in creatinine tubular secretion (that approximately accounts for $15 \%$ of total creatinine clearance); studies on the proximal tubulus revealed that this is the real target of TDF toxicity with urinary loss of low molecular weight proteins (LMWPs).

A meta-analysis including more than 5000 subjects showed larger decreases in estimated creatinine clearance $(3.92 \mathrm{ml} / \mathrm{min}$; $95 \% \mathrm{CI}$ : 2.13-5.70) in TDF-treated patients [6]. Chronic kidney disease was predicted by older age, intravenous drug use, Hepatitis $\mathrm{C}$ virus (HCV) co-infection, lower baseline estimated glomerular filtration rate (eGFR), female gender, lower nadir CD4 count, hypertension, diabetes and cardiovascular disease [7]. Notably, every year of TDF exposure was associated with increased hazard ratios for proteinuria (1.30 [95\% CI: 1.22-1.37]), rapid decline in kidney function (1.17 [95\% CI: 1.11-1.24]) or chronic kidney disease (1.44 [95\% CI: 1.30-1.60]) and such conditions were only partially reversible [8].

In several observations the concomitant use of ritonavir-boosted protease inhibitors (bPIs) was associated with the highest risk of renal and
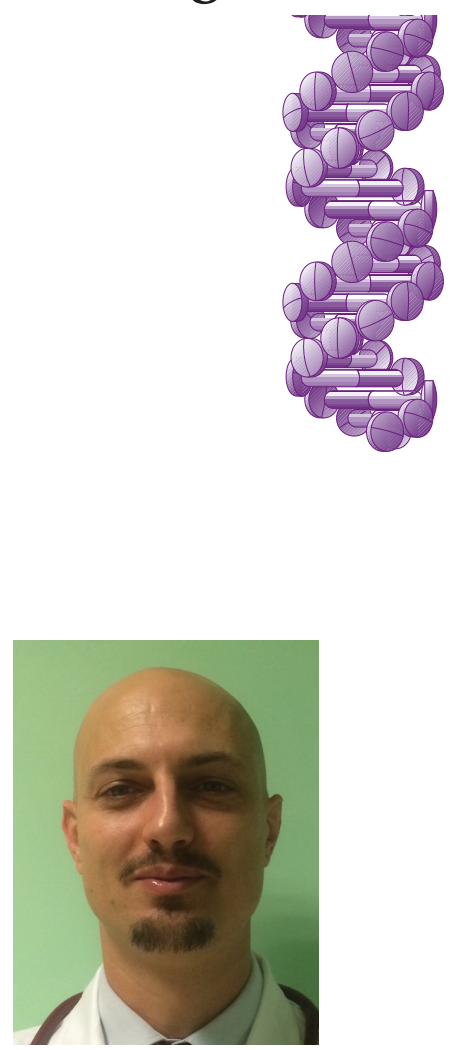

Andrea Calcagno Author for correspondence: Unit of Infectious Diseases, Department of Medical Sciences, University of

Torino, Torino, Italy

Tel.: +390114393884

Fax: +390114393942

andrea.calcagno@unito.it

Giovanni Di Perri

Unit of Infectious Diseases, Department of Medical Sciences, University of Torino, Torino, Italy

Stefano Bonora

Unit of Infectious Diseases, Department of Medical Sciences, University of Torino, Torino, Italy

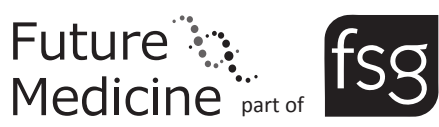


bone toxicity. The co-administration of either boosted or unboosted protease inhibitors leads to a greater initial decline in eGFR than efavirenz: this decline was higher with atazanavir/ritonavir than with lopinavir/ritonavir [9]. Notably atazanavir has been associated with nephrolithiasis and, although in few case reports, with interstitial nephritis, thus suggesting the potential independent nephrotoxicity of the compound [10]. The use of bPIs in patients with a high baseline risk profile was associated with a low number needed to harm at 5 years (21 and nine patients according to the compound), thus suggesting caution in selected group of patients [7].

"...the association of TDF with protease inhibitors
or cobicistat-boosted drugs should be carefully
evaluated and alternative treatments considered."

TFV exposure has been found to be associated with renal toxicity: higher TFV plasma concentrations have been observed in patients with proximal tubular dysfunction or reduced eGFR [11]. The combined effect of higher plasma exposure and longer duration of TDF use was recognized as the best predictor of tubular impairment (defined using 24-h urine analysis) [12]. A recent study by our group observed a reduced TFV clearance in patients with subclinical tubular dysfunction defined by abnormally elevated LMWPs in spot urines: this provides a simple method for estimating TFV clearance in vivo [13].

Several genetic polymorphisms in regions coding for efflux transporters have been associated with TDFassociated tubular toxicity and they have been recently reviewed [4]. ABCC2 (encoding for MRP-2) SNPs have been associated with tubular toxicity; nevertheless these observations are difficult to rationalize because tenofovir is not a substrate for MRP-2 although an indirect effect may not be excluded [14]. A study in HIV-positive patients found that an ABCC4 (encoding for MRP-4) SNP (669 C>T, rs899494) was associated with renal toxicity and to treatment discontinuation (along with low body weight) [15]. In patients on TDF, two ABCC10 polymorphisms (encoding for MRP-7) (526 G>A, rs9349256 and $2843 \mathrm{~T}>\mathrm{C}$, rs2125739) were associated with kidney toxicity [16] but replication studies are lacking. A recent study comparing patients with TDF-induced Fanconi's syndrome to TDFtreated controls revealed that several rare variants in $A B C C 2$ and Lowe syndrome protein OCRL gene were associated with decreased eGFR [17].

\section{Factors associated with TFV exposure \& clearance}

Several of the above highlighted risk factors also affect TFV pharmacokinetics and therefore this might be an optimal scenario for TDF dose tailoring given the observed concentration-dependent effect.

Low body weight (and low weight/serum creatinine), older age and lower eGFR have been associated with increased TFV exposure [18,19]. Protease inhibitors (PI) co-administration (both boosted and unboosted) is the commonest factor associated with higher TFV plasma concentrations [20]. The mechanism by which boosted PIs increase TDF exposure (20-35\%) is thought to be dependent upon inhibition of intestinal p-glycoprotein intestinal inhibition with increased absorption; their inhibitory effect on MRP-2 and MRP-4 is less clear. The effect of cobicistat seems similar to the one observed with ritonavir with increased TFV absorption; the additional inhibitory effect on MATE-1 results in a harmless 'cosmetic' serum creatinine elevation (by decreased tubular secretion) with no reduction in actual filtration rates.

While the effect of SNPs in transporter encoding genes on TFV exposure is still debated, some associations have been observed. An ABCC4 SNP (4131 T>C, rs3742106) has been associated with increased intracellular concentrations of TFV triphosphate $(35 \%$ higher, using human peripheral blood mononuclear cells) suggesting a similar effect in tubular cells. In another study eGFR, bPIs co-administration and SLC28A2 (encoding for concentrating nucleoside transporter 2) CT/TT genotypes (124 C>T, rs11854484) were independently associated with plasma TFV exposure; it should be however highlighted that so far no study confirmed that TFV is a concentrating nucleoside transporter-2 substrate [21]. In the same study $A B C C 10$ SNPs $(1791+526 \mathrm{G}>\mathrm{A} 138$, rs9349256) and bPIs co-administration were independently associated with TFV clearance (as measured by urinary to plasma 12-h concentration ratio).

\section{Conclusion: tailoring TDF administration}

The pathogenesis of renal damage warrants studies aiming at reducing TFV exposure and/or its entrapment into tubular cells. It is already recommended to decrease TDF frequency of administration in patients with reduced estimated glomerular filtration: with eGFR in the $30-49 \mathrm{ml} / \mathrm{min}$ range, a every other day administration of a $300 \mathrm{mg}$ tablet is suggested. A recent study in Thai patients supported the equivalence of TDF $150 \mathrm{mg}$ every day to the standard $300 \mathrm{mg}$ dose in a group of renally impaired patients [22]. Apart from this study no reports on TDF dose reduction have been published; however the promising results of a ' 5 days on/2 days off' strategy with efavirenz/TDF/emtricitabine support a less frequent administration of TDF in stable and adherent patients [23]. This approach is further endorsed by the 
long intracellular half-life of TFV diphosphate, which is about $120 \mathrm{~h}$.

The pharmacology of TAF, associated with $86.1 \%$ lower TFV plasma but higher intra-lymphocyte concentrations, possibly accounts for its favorable renal and bone safety profile. These properties fit with the improved tubular safety recorded in a small case series of HIV/HBV co-infected patients with previous severe tubular damage, in whom probenecid (an organic anion transporter 1 inhibitor, probably limiting TFV uptake by tubular cells) was added to the TDF-containing regimen.

Waiting for the results of prospective studies assessing the efficacy and safety of TDF reduced doses when associated with protease inhibitors (as recently envisaged) [24] and for the long-term studies on TAF safety and compartmental efficacy, a personalized approach to TDF might be used. Although the pathogenic link between subclinical tubular dysfunction and reduced bone mineral density is unclear, the recent observation of lower BMD in patients with high urinary retinol binding protein (corrected for urinary creatinine) suggests caution in certain high-risk groups. The specific monitoring of tubular function (either through 24-h urine analysis or through LMWPs on spot urines) might be envisaged given the available alternatives.

In specific high-risk groups including subjects with low BMI, postmenopausal females, patients aged $>65$ years and those with reduced eGFR or renalaffecting concomitant diseases (including diabetes, hypertension and kidney stones or renal abnormalities),

\section{References}

1 Ustianowski A, Arends JE. Tenofovir: what we have learnt after 7.5 million person-years of use. Infect. Dis. Ther. 4(2), 145-157 (2015).

2 Tourret J, Deray G, Isnard-Bagnis C. Tenofovir effect on the kidneys of HIV-infected patients: a double-edged sword? J. Am. Soc. Nephrol. 24(10),1519-1527 (2013).

3 Uwai Y, Ida H, Tsuji Y, Katsura T, Inui K. Renal transport of adefovir, cidofovir, and tenofovir by SLC22A family members (hOAT1, hOAT3, and hOCT2). Pharm. Res. 24, 811-815 (2007).

4 Moss DM, Neary M, Owen A. The role of drug transporters in the kidney: lessons from tenofovir. Front. Pharmacol. 5 , 248 (2014).

5 Ramamoorthy H, Abraham P, Isaac B. Mitochondrial dysfunction and electron transport chain complex defect in a rat model of tenofovir disoproxil fumarate nephrotoxicity. J. Biochem. Mol. Toxicol. 28, 246-255 (2014).

6 Cooper RD, Wiebe N, Smith N, Keiser P, Naicker S, Tonelli M. Systematic review and meta-analysis: renal safety of tenofovir disoproxil fumarate in HIV-infected patients. Clin. Infect. Dis. 51(5), 496-505 (2010). the association of TDF with protease inhibitors or cobicistat-boosted drugs should be carefully evaluated and alternative treatments considered.

Recent international guidelines suggest the use of integrase inhibitor-based combination treatments in naive patients thus reducing the potential detrimental effect of PI co-administration. In this group of previously untreated subjects antiretroviral efficacy is the main objective of treatment and short-term toxicity less a concern thus advising the use of standard TDF dose.

In stable patients with durable suppression of viral replication under ritonavir or cobicistat-boosted antiretroviral treatments, the presence of another risk factor (including $\mathrm{BMI}<20 \mathrm{~kg} / \mathrm{m}^{2}$, age $>65$ years or postmenopausal state) might warrant the use of TDF $300 \mathrm{mg}$ every other day. Whether the use of pharmacokinetics ( $A B C C 10$ and $A B C C 4$ SNPs) could be cost-effective for avoiding unnecessary toxicity to patients with less common variants need to be prospectively evaluated.

\section{Financial \& competing interests disclosure}

A Calcagno received research grants from Bristol-Myers Squibb and Gilead. A Calcagno, G Di Perri and S Bonora received consultancy fees from Abbvie, Bristol-Myers Squibb and Gilead and speaker's honoraria from Janssen-Cilag, Merck-Sharp \& Dohm and Viiv. The authors have no other relevant affiliations or financial involvement with any organization or entity with a financial interest in or financial conflict with the subject matter or materials discussed in the manuscript apart from those disclosed.

No writing assistance was utilized in the production of this manuscript.

7 Mocroft A, Lundgren JD, Ross M et al. Development and validation of a risk score for chronic kidney disease in HIV infection using prospective cohort data from the D:A:D study. PLoS Med. 12(3), e1001809 (2015).

8 Scherzer R, Estrella M, Li Y et al. Association of tenofovir exposure with kidney disease risk in HIV infection. AIDS 26(7), 867-75 (2012).

9 Young J, Schäfer J, Fux CA et al. Renal function in patients with HIV starting therapy with tenofovir and either efavirenz, lopinavir or atazanavir. AIDS 26(5), 567-75 (2012).

10 Kanzaki G, Tsuboi N, Miyazaki Y, Yokoo T, Utsunomiya Y, Hosoya T. Diffuse tubulointerstitial nephritis accompanied by renal crystal formation in an HIV-infected patient undergoing highly active antiretroviral therapy. Intern. Med. 51(12), 1543-1548 (2012).

11 Poizot-Martin I, Solas C, Allemand J, Obry-Roguet V, Pradel $\mathrm{V}$, Bregigeon $\mathrm{S}$ et al. Renal impairment in patients receiving a tenofovir-cART regimen: impact of tenofovir trough concentration. J. Acquir. Immune Defic. Syndr. 62, 375-380 (2013).

12 Ezinga M, Wetzels JF, Bosch ME, van der Ven AJ, Burger DM. Long-term treatment with tenofovir: prevalence of 
kidney tubular dysfunction and its association with tenofovir plasma concentration. Antivir. Ther. 19, 765-771 (2014).

13 Calcagno A, Cusato J, Marinaro L et al. Tenofovir clearance is reduced in hiv-positive patients with subclinical tubular impairment. AIDS PMID: 26636928 (2015) (Epub ahead of print).

14 Nishijima T, Komatsu H, Higasa K et al. Single nucleotide polymorphisms in $A B C C 2$ associate with tenofovir-induced kidney tubular dysfunction in Japanese patients with HIV-1 infection: a pharmacogenetic study. Clin. Infect. Dis. 55, 1558-1567 (2012).

15 Lubomirov R, Colombo S, di Iulio J et al. Association of pharmacogenetic markers with premature discontinuation of first-line anti-HIV therapy: an observational cohort study. J. Infect. Dis. 203, 246-257 (2011).

16 Pushpakom SP, Liptrott NJ, Rodríguez-Nóvoa S et al. Genetic variants of $A B C C 10$, a novel tenofovir transporter, are associated with kidney tubular dysfunction. J. Infect. Dis. 204, 145-153 (2011).

17 Dahlin A, Wittwer M, de la Cruz M et al. A pharmacogenetic candidate gene study of tenofovir-associated Fanconi syndrome. Pharmacogenet. Genomics 25(2), 82-92 (2015).

18 Gervasoni C, Meraviglia P, Landonio S et al. Low body weight in females is a risk factor for increased tenofovir exposure and drug-related adverse events. PLoS ONE 8(12), e80242 (2013).
19 Baxi SM, Greenblatt RM, Bacchetti P et al. Common clinical conditions - age, low BMI, ritonavir use, mild renal impairment - affect tenofovir pharmacokinetics in a large cohort of HIV-infected women. AIDS 28(1), 59-66 (2014).

20 Calcagno A, Gonzalez de Requena D, Simiele M et al. Tenofovir plasma concentrations according to companion drugs: a cross-sectional study of HIV-positive patients with normal renal function. Antimicrob. Agents Chemother. 57(4), 1840-1843 (2013).

21 Calcagno A, Cusato J, Marinaro L et al. Clinical Pharmacology of tenofovir clearance: a pharmacokinetic/ pharmacogenetic study on plasma and urines. Pharmacogenomics J. PMID: 26440731 (2015) (Epub ahead of print).

22 Cressey TR, Avihingsanon A, Halue G et al. Plasma and intracellular pharmacokinetics of tenofovir disoproxil fumarate $300 \mathrm{mg}$ every 48 hours vs $150 \mathrm{mg}$ once daily in HIV-infected adults with moderate renal function impairment. Clin. Infect. Dis. 61(4), 633-639 (2015).

23 Reynolds SJ, Kityo C, Hallahan CW et al. A randomized, controlled, trial of short cycle intermittent compared with continuous antiretroviral therapy for the treatment of HIV infection in Uganda. PLoS ONE 5(4), e10307 (2010).

24 Hill A, Khoo S, Back D, Pozniak A, Boffito M. Should the dose of tenofovir be reduced to $200-250 \mathrm{mg} /$ day, when combined with protease inhibitors? J. Int. AIDS Soc. 17(4 Suppl. 3), 19583 (2014). 